\title{
Política educativa para migrantes en Chile: un silencio elocuente
}

\author{
María Loreto Mora Olate \\ Universidad del Bío-Bío-CONICYT, Chillán, Chile. \\ Email:mlmora@ubiobio.cl
}

\begin{abstract}
Resumen: Se plantea cómo primer objetivo, proponer una mirada crítica de las políticas educativas vigentes en Chile, desde la clave de lectura referida al proceso deescolarización de migrantes, en términos de desentrañar cómo es abordado en diversos instrumentos curriculares, ejercicio que permite develar quiénes han sido los cómplices de ese "silencio". Como segundo objetivo, esta reflexión pretende argumentar que dicha ausencia de una política de escolarización de niños y jóvenes migrantes, podría constituirse en una oportunidad para fortalecer procesos de profesionalidad docente y en un desafío político educativo en la atención de la diversidad cultural desde una perspectiva de educación intercultural crítica. crítica.

Palabras clave: Migrantes, política educativa, profesorado, interculturalidad
\end{abstract}

\section{Educational policy for migrants in Chile: an eloquent silence}

\begin{abstract}
As a first objective, a critical view of the current educational policies in Chile is proposed, from a position referred to the schooling process for migrants, in terms of revealing how it is approached among different curricular instruments, an exercise that tells who have been the accomplices of that "silence". As a second objective, this reflection intends to argue that the absence of a schooling policy for migrant children and young people could constitute an opportunity to strengthen processes of teacher professionalism and a political educational challenge in the attention of cultural diversity, from a perspective of critical intercultural education.
\end{abstract}

Keywords: Migrants, educational policy, teachers, critical interculturalism

\section{A Política educacional para migrantes no Chile: um silêncio eloquente}

Resumo: Como primeiro objetivo propõe-se uma visão crítica das políticas educativas vigentes no Chile. Para isso, será utilizado como ponto chave a leitura referente ao processo de escolarização de migrantes, como uma forma de desvendar sua abordagem em vários instrumentos curriculares, exercício que permite revelar quem foram os cúmplices desse "silêncio". Como segundo objetivo, esta reflexão visa também argumentar que a ausência de uma política escolar para crianças e jovens migrantes, poderia ser uma oportunidade de fortalecimento dos processos de profissionalismo docente e de um desafio político educativo, atentando para a diversidade cultural com base em uma perspectiva de educação intercultural crítica. 
Palavras-chave: Migrantes, política educacional, professores, interculturalidade crítica.

\section{Introducción}

Desde el regreso de la democracia en el año noventa, Chile viene experimentando con fuerza el fenómeno de la migración, debido a su crecimiento económico y estabilidad política, resultando un destino atractivo especialmente para los países de la región. Es así como de ser solo un país emisor depersonas migrantes, ahora se ha transformado en uno receptor, pero sin contar aún con una política migratoria (Thayer, 2016) acorde con los tipos de migraciones internacionales.

En el plano investigativo latinoamericano, "la escolarización de niños y jóvenes migrantes es un tema que no se ha convertido en un campo de investigación” (Hernández,2016, p.152), según lo documentado por el autor tomando datos de la OEA (2011), explica que el foco recurrente en la investigación académica transita en temas como el tráfico, la explotación, la reunificación familiar o "la integración general de la infancia migrante, donde entra la educación como un tema secundario” o bajo el amparo de la políticas públicas relacionadas con diversidad étnica (Ibid).

Entonces no resulta del todo paradojal yuxtaponer a "silencio" el concepto de "elocuencia”, cuyo significado remite a "eficacia para persuadir o conmover que tienen las palabras, los gestos o ademanes y cualquier otra acción o cosa capaz de dar a entender algo con viveza” (RAE, 2014), porque permite que la tesis del silencio elocuente, transite por dos vías, la primera, evidencia el desfase de la política pública frente a escenarios crecientes de diversidad cultural que se viven en las escuelas en Chile, permaneciendo hasta hoy silente; y la segunda, representa una oportunidad para devolver la voz a los docentes bajo un paradigma intercultural crítico que tiene como horizonte ideal la construcción de una escuela intercultural, favorecedora de "una reflexión crítica sobre el conflicto propio del contacto entre culturas y enseña a comprender el desarrollo de los pueblos en el marco de relaciones históricas de dominación y vasallaje, legitimando la alteridad en múltiples escenarios” (Hevia, 2005,p.600).

La clave del vals, “Si vas para Chile” ${ }^{1}$ compuesto por Chito Faró (1942), escenario del mito que configura el carácter hospitalario de chileno, resumido en el verso: "Y verás cómo quieren en Chile al amigo cuando es forastero”, mito que se triza al contrastarlo con el fenómeno de la migración, debido a la situación que viven los migrantes que llegan a Chile, que dista bastante de una historia de amor. En el país rige una Ley migratoria que data del año 1975 (Ley 1094) que concibe al extranjero y a la migración como una amenaza, conforme a su contexto dictatorial de producción, que incorpora elementos de la Doctrina de Seguridad Nacional, sustentada en la amplia 
discrecionalidad, fuerte control de fronteras y amenaza (Sandoval, 2016). Evidentemente, este cuerpo legal no entrega respuestas para las actuales exigencias que imponen los flujos migratorios, ya que se aleja del enfoque de derechos y de los acuerdos internacionales adscritos por Chile en esa materia. Si bien dentro del programa de gobierno de la presidenta Bachelet se incorpora el compromiso de reformar la legislación migratoria, el trabajo legislativo no ha tenido la agilidad esperada.

La cadencia del vals símbolo de la hospitalidad, da paso a los sones del rock del grupo chileno "Los Prisioneros", con su tema Maldito sudaca (González,1987), donde se escucha fuerte: "horrible boliviano, vil ecuatoriano/cochino sudaca, cochino latino”, siendo la ironía denunciante quien termina de trizar el mito de Chile como una nación hospitalaria, develando rasgos de racismo y xenofobia en diferentes planos sociales y en lo específico, el educativo que es foco de interés para esta reflexión.

Es así como a través de una revisión documental,se plantea como primer objetivo, proponer una mirada crítica de las políticas educativas vigentes en Chile, desde la clave de lectura referida al proceso de escolarización de migrantes, en términos de responder a cómo es abordado en diversos instrumentos legales y curriculares, ejercicio que con el respaldo de evidencia investigativa, permite develar quiénes han sido los cómplices de ese "silencio"; análisis desarrollado en la primera parte del texto. A continuación, como segundo objetivo, sepretendeargumentar que dicha ausencia de una política de escolarización de niños y jóvenes migrantes, podría constituirse en una oportunidad para fortalecer procesos de profesionalidad docente y en un desafío político educativo en la atención de la diversidad cultural desde una perspectiva de educación intercultural crítica.

Chile al no contar con una legislación migratoria moderna y tampoco con una política de educación y migración, revelaría“la existencia de un Estado negligente ante una población migrante en condición de exclusión social, y una ciudadanía nativa carente de herramientas para enfrentar en su vida cotidiana esta nueva realidad" (Thayer,2016, p.71). Entonces el "mito de la hospitalidad” del chileno viene a encubrir más bien la subordinación hacia "el otro" de rasgos europeos y con pensamiento occidental, aquel llamado "extranjero", porque al afuerino latinoamericano se le denomina "migrante", cayendo en un ejercicio de menosprecio hacia nuestra identidad mestiza y de deslegitimación de nuestros saberes. Conforme al actual escenario, y parafraseando el vals de Chito Faró, el verso debería mutar a: "Y verás cómo quieren en Chile, al amigo cuando es extranjero europeo, blanco y rubio”.

\section{Cómplices del silencio}

El contexto escolar es un espacio fundamental para posibilitar la incorporación de los migrantes en la sociedad que los acoge y en esta tarea los docentes adquieren un rol clave en el proceso de socialización y recep- 
ción de estos estudiantes, actuación que responde a los imperativos impuestos por las políticas educativas del Estado chileno.

Desde la categoría analítica escolarización de migrantes, este apartado se propone una revisión documental crítica de las políticas educativas vigentes en Chile, en términos de cómo es abordada dicha realidad en los diversos instrumentos (“cómplices del silencio”),que concretan la política en diferentes niveles. En ellos, es posible constatar contradicciones y ausencias en relación con la escolarización de niños y jóvenes migrantes, a pesar de los compromisos asumidos con la comunidad internacional, a partir del año 1990, enfocados a velar por los derechos de los niños y niñas (Palma, 2015, p.4) y de la declaración en la Constitución Política, que garantiza el acceso a la educación para todos los/as niños, niñas y jóvenes que residen en nuestro territorio. Sin embargo, la carta fundamental desde una concepción de estado unitario no alude la realidad multicultural del territorio nacional, entonces el derecho a la educación parece quedar reducido al acceso, pero no a las condiciones de ella. Al mismo tiempo, esto nos indica una incongruencia con la Convención sobre los Derechos del niño (Congreso de Chile, Decreto supremo N830. 14 de agosto de 1990), donde Chile, como uno de los Estados Partes, se compromete a respetar el derecho de los niños y las niñas a preservar su identidad cultural y los valores nacionales de su país originario (Artículo 8 y 29, letra c).

Por tanto, la vetusta Ley de inmigración chilena, unido a la poca celeridad del trabajo legislativo y por consiguiente, la ausencia del políticas migratorias integrales, serían a nivel general, los marcos contextuales cómplices de la ausencia de una política educacional de escolarización de migrantes; escenario donde la diversidad cultural migrante se hace cada día más presente en las aulas de nuestro país.

En un nivel más particular, es necesario reconocer otro antecedente confabulador: las políticas educativas que dieron forma la reforma educacional de los noventa en Chile. Al respecto y de acuerdo a la argumentación entregada por Oliva (2008, p.220), la reforma curricular de los noventa es heredera de su homónima del año 1965, la cual instala un modelo curricular técnico "que se dota de atributos de “objetividad” y "neutralidad”, que enmascaran su ideología, siendo una manifestación de la continuidad de nuestra condición colonial, por lo tanto, la reforma del currículum “colaboró a la profundización de la desigualdad educativa, al fracturar profundamente el carácter moral de lo educativo” (Ibid). Uno de los agentes más golpeados fueron los docentes, que debieron asumir su rol desde una racionalidad técnica, “cercenándoles sus posibilidades de formar una comunidad reflexiva”, recibiendo un golpe fatal, viéndose limitados para ejercer su rol de intelectuales, en sus dimensiones éticas y políticas de su función profesional (Oliva,2008). Por su parte, Donoso y Donoso (2009), al realizar un balance de los veinte años de las políticas de gestión de la educación pública y escolar en Chile (1990-2010) develan la pugna entre dos paradigmas, que generaron, por un lado, políticas propias del Estado de Bienestar y por otro, políticas de mercado. El efecto de esta lucha es que las políticas educativas 
no han tenido un impacto en la mejora de los aprendizajes (Donoso y Donoso, 2009, p. 429).

Una vez realizada esta contextualización, la propuesta de lectura crítica de la política educativa vigente en Chile, arroja los siguientes cómplices del silencio, que se detallan en cada caso como sigue:

\section{Marco legal regulatorio de la educación}

-La Ley General de Educación(Congreso de Chile, Ley Nº 20.370, 17 de agosto de 2009), define a la educación como:

el proceso de aprendizaje permanente que abarca las distintas etapas de la vida de las personas y que tiene como finalidad alcanzar su desarrollo espiritual, ético, moral, afectivo, intelectual, artístico y físico, mediante la transmisión y el cultivo de valores, conocimientos y destrezas. Se enmarca en el respeto y valoración de los derechos humanos y de las libertades fundamentales, de la diversidad multicultural y de la paz, y de nuestra identidad nacional, capacitando a las personas para conducir su vida en forma plena, para convivir y participar en forma responsable, tolerante, solidaria, democrática y activa en la comunidad, y para trabajar y contribuir al desarrollo del país(LGE, 2009).

Esta ley establece que ni el estado, ni los establecimientos educacionales podrían discriminar arbitrariamente a los/as estudiantes y a los demás miembros de la comunidad educativa. Por tanto, los/as niños/a y jóvenes migrantes, no verían afectados este derecho, aun cuando sus padres no tengan regularizada su residencia en el país, porque estos estudiantes "deberán ser aceptados y matriculados provisionalmente en los establecimientos educacionales" y se les considerará "como alumno/a regular para todos los efectos académicos, curriculares y legales, teniendo el sostenedor derecho a la subvención correspondiente". A su vez, el Ministerio señala que "los/as alumnos/as inmigrantes tienen los mismos derechos que los nacionales: seguro escolar, pase escolar, becas y alimentación, entre otros beneficios”. Dicha repartición gubernamental, también explica claramente en su sitio web el procedimiento a seguir para obtener una matrícula provisoria o definitiva (Mineduc,2016a).

-Ley de inclusión escolar (Congreso de Chile, Ley N² 20. 845, 8 de junio de 2015), pone fin al lucro en la educación con recursos públicos, propone un nuevo sistema de admisión y establece la gratuidad progresiva de la educación, mediante el aumento de recursos destinados a calidad. El fin de la selección apunta a que los establecimientos deberán aceptar a todos los y las postulantes, si disponen de vacantes. Bajo esta ley, el sistema propenderá a "eliminar todas las formas de discriminación arbitraria que impidan el aprendizaje y a participación de los y las estudiantes. Asimismo, el sistema propiciará que los establecimientos educativos sean un lugar de 
encuentro entre las y los estudiantes de distintas condiciones socioeconómicas, culturales, étnicas, de género, de nacionalidad o de religión (Art.1, numeral 1, letra e).

Se aspira avanzar desde el modelo de integración hacia el de la inclusión, que propone una transformación de las culturas, políticas y prácticas de las instituciones escolares en función de las características de los estudiantes, donde la diversidad es "una condición transversal a los seres humanos, y, por lo tanto, los procesos educativos requieres flexibilizarse, de modo de ser pertinentes a esta diversidad” (Mineduc, 2016b, p.13). Se deriva entonces, que este enfoque educativo inclusivo, pretende desmontar la construcción homogénea y normalizada de estudiante. No obstante, el espíritu de estas dos leyes resulta tensionado ya sea en términos del acceso y de la permanencia en el sistema educativo del estudiante migrantes, donde el paradigma desde el cual “se interpreta la relación con el “otro” es el de la integración por sobre el ideal de inclusión propuesto desde la política pública” (Bustos y Gairín, 2017, p. 210). El Marco legal regulatorio de la educación, expresado en la LEGE, asegura el acceso al sistema escolar, pero no las condiciones de permanencia y acceso a beneficios, como los provenientes de la Junta Nacional de Auxilio Escolar y Becas (Junaeb) o de la Ley de Subvención Escolar Preferencial , debido al "Rut 100”, asignado por el Mineduc provisoriamente estudiantes en situación migratoria irregular, quienes son admitidos en el sistema escolar, "pero en el ejercicio de su derecho a la educación están condicionados a la obtención de una Visa y, por lo tanto, a un RUN definitivo” (eligeeducar.cl). Esta situación los/as imposibilita, por ejemplo, para rendir la Prueba de Selección Universitaria (PSU), o contar con NEM y Ranking de Notas para el ingreso a la educación superior; también los/as limitaen su acceso a los beneficios de apoyo escolar, útiles y becas de alimentación (JUNAEB) y a los programas de apoyo pedagógico (PACE, PIE, SEP) y no obtienen su certificado de estudios por el MINEDUC; por lo tanto, su trayectoria escolar no es reconocida.

Como una forma de solucionar dicha situación, el Ministerio de Educación anuncia en noviembre del año 2016, la creación del Identificador Provisorio Escolar (IPE) para estudiantes migrantes, que permitirá acceder a dichos beneficios (Mineduc, 2016a ).Sin embargo, el IPE permite acceder a cuatro de los diecisiete beneficios de la Juaneb: alimentación, textos escolares y computadores del programa “Me conecto para aprender”, no pudiendo ser beneficiarios de aquellas ayudas que requieren la obtención del RUN (Rol Único Nacional) y cédula de identidad (Universidad Diego Portales, 2017).La evidencia investigativa también nos ayuda a evidenciar que la ley "es letra muerta”:

(...) los colegios, y los funcionarios de estos, tienen diferentes posturas (explícitas o implícitas) en relación a la recepción de estudiantes extranjeros; unas más cercanas a los planteamientos del derecho universal a la educación y a la valoración de la presencia de extranjeros en las aulas; otras lamentablemente más cercanas, en mayor o menor medida, a la discriminación hacia estos grupos. La evidencia 
cualitativa nos muestra que muchas veces, de estas posturas va a depender al fin y al cabo del nivel de acogida y buena atención que tenga un apoderado extranjero en busca de matrícula. A muchos apoderados extranjeros de grupos socioeconómicos bajos o medio bajo, se les ha negado a priori la matrícula para sus hijos por razones no justificadas, esto se da por medio de diferentes mecanismos entre los que encontramos: la falsa "falta de matrícula”, solicitud de documentos o requisitos fuera de la norma y abierta discriminación, "no recibimos extranjeros” (Stefoni, Acosta, Gaymer\& Casas-Cordero, 2008, p. 25).

En resumen, los y las escolares migrantes han estado atrapados en la incongruencia de las leyes chilenas, las cuales no habrían estado respetando acuerdos internacionales como los Derechos de los niños y las niñas, vulnerando así sus derechos humanos.

\section{Marco Curricular}

Las Bases curriculares de la Educación Parvularia (Mineduc, 2005), en sus orientaciones valóricas, concibe a los niños y las niñas insertos en una comunidad comprometida con ellos y como un espacio de aprendizaje y de "acogida de todos y cada uno de sus miembros, el respeto y la valoración por la diversidad, los distintos roles que desempeñan las personas e instituciones, la solidaridad, la resolución pacífica de conflictos, la participación democrática y la construcción de ciudadanía”.

Destaca también la consignación de respetar y valorar la diversidad étnica, lingüística y cultural de las diferentes comunidades que conforman el país, realidades que se hacen "necesarias de reconocer e incorporar en la construcción e implementación curricular” (Mineduc, 2005,p.13). No obstante, en la práctica educativa, existe evidencia que indica la implementación de un currículo de carácter hegemónico, que reproduce un patriotismo exacerbado en este nivel educativo a través de la ritualidad de los actos cívicos que escenifican el nacionalismo soslayando el contexto de diversidad cultural transfronteriza del norte de Chile(Mondaca, Rivera \& Gajardo, 2013).

Las Bases curriculares para la Educación Básica (Mineduc, 2012b), cumplen el rol de ofrecer una "base cultural común para todo el país, mediante Objetivos de aprendizaje establecidos para cada curso o nivel" (Mineduc, 2012b, p.16), lo cual persigue asegurar que todos los alumnos accedan a "una educación de calidad con igualdad de oportunidades" (Mineduc, 2012b, p.18).Entre los objetivos generales planteados para este nivel educativo, referidos al ámbito personal y social, destaca la aspiración a que los/as niños/as reconozcan y respeten "la diversidad cultural, religiosa, y étnica y las diferencias entre las personas, así como la igualdad de derechos entre hombres y mujeres”, y que desarrollen capacidades de empatía con otros (Mineduc,2012b, p.14). Por su parte, en los Objetivos de Aprendizaje Transversales, en el ámbito correspondiente a la Dimensión 
Moral, se establece como eje a los Derechos Humanos, referente que orienta la conducta personal y social. Esasí como uno de los objetivos declara que los y las estudiantes deberán: “conocer, respetar y defender la igualdad de derechos esenciales de todas las personas, sin distinción de sexo, edad, condición física, etnia, religión o situación económica, y actuar en concordancia con el principio ético que reconoce que todos los seres humanos nacen libres e iguales en dignidad y derechos (....)” (Mineduc, 2012 b) p.28).

No obstante a lo consignado, las Bases curriculares tienden a legitimar un currículum hegemónicoque tiende a excluir los referentes culturales bajo un concepto de igualdad mal entendido y que profundiza procesos de normalización, donde "la situación de los alumnos migrantes no es un tema de reflexión para los docentes” (Bustos \& Gairín, 2017, p. 200); es decir, se ejerce una exclusión curricular al ignorar las culturas presentes en la sociedad(Torres, 2012).Si bien en el Marco curricular es repite la palabra "diversidad", donde es posible leerla en expresiones como "diversidad cultural”, "diversidad étnica”, “diversidad humana”, este uso retórico queda atrapado en el enfoque técnico por competencias, que transversaliza la educación desde el nivel parvulario hasta la educación superior.

La hegemonía monocultural difícilmente ha resultado permeada, ya que la construcción de currículos interculturales es aún una fantasía, donde "la contextualización del mismo se traduce en un esfuerzo individual de algunos docentes por trabajar contenidos étnicos, pero la base cultural de referencia del currículo permanece inalterable y sin discusión” (Hevia,2005, p.612). De esta forma, la gestión de la diversidad cultural migrante por parte del sistema educativo entraña una discriminación indirecta, específicamente en materia curricular donde las Bases Curriculares de Historia, Geografía y Ciencias Sociales "están sustentadas en un enfoque tradicional y conservador, con una mayoría de objetivos de aprendizaje que hacen referencia a la historia o al territorio chileno, no reconociendo los escenarios de multiculturalidad presentes en las aulas" (Universidad Diego Portales, 2017, p. 350).

Recién en la Política Nacional de Desarrollo Curricular (Mineduc, 2016c), se realiza un reconocimiento de la realidad pluricultural, plural y diversa en distintos sentidos de nuestra sociedad chilena, conforme al momento actual de Reforma Educacional, donde se observa una preocupación por generar un ordenamiento, una estabilidad curricular, porque cada gobierno de turno ha instaurado cambios que toman más años de los que dura un mandato, lo cual ha provocado un desfase en la implementación y apropiación de las nuevas Bases curriculares. Es así como, el Ministerio de Educación ha considerado "fortalecer una política curricular de Estado, con perspectiva país y de largo plazo, para el desarrollo curricular nacional, en que se resguarde la pertinencia, relevancia, contextualización, legitimidad y estabilidad del currículum para el sistema educativo” (Mineduc, 2016 c) p.4). Producto del trabajo de la mesa de Desarrollo Curricular, se generaron recomendaciones para los cinco grandes ámbitos del sistema curricular actual: 
-Orientaciones generales para el currículum nacional: la mesa de trabajo comparte la idea que "la sociedad chilena es pluricultural, plural y diversa en distintos sentidos. Esto es esencial para una sociedad democrática que reconoce y valora las diferencias, a la vez que promueve la igualdad de derechos de todos sus integrantes. Entonces, el currículum nacional debe sustentarse en "el reconocimiento de la pluriculturalidad del país y, por lo tanto, debe ser desarrollado considerando un diálogo armónico entre las perspectivas local, nacional y global” En este sentido, el currículum nacional debe partir de la base que los pueblos indígenas existentes en el país “son portadores de culturas específicas y, en consecuencia, es necesario reconocer que la diversidad y pluriculturalidad constituyen la identidad nacional” (Mineduc, 2016c, p. 21). Al mismo tiempo, se establece que las lógicas de construcción de un currículum nacional ha de ser "pensado desde una perspectiva de inclusividad fundada en una comprensión compleja de la diferencia, que no busque normalizarla o asimilarla. Esto implica una prescripción curricular flexible, relevante y pertinente, para el despliegue de la máxima potencialidad de la diversidad de estudiantes (Mineduc, 2016c, p.21).

-Procesos de producción del currículum: deben considerar criterios de diversidad, pertinencia y validez.

-Implementación y desarrollo curricular: la Mesa entiende que los docentes y las comunidades educativas deben tener un rol activo en relación con la prescripción curricular, expresadas en las capacidades de reflexión y análisis del currículum nacional, “que permitan desarrollar la comprensión de sus propósitos y contenidos en consonancia con intervenciones pedagógicas pertinentes y consistentes” (Mineduc, 2016c, p.30). de Calidad.

-Evaluación curricular: que también considere los Otros indicadores

-Institucionalidad: referido al monitoreo e interpretación de los cambios que se producen en la "sociedad (nacional e internacional) y en el sistema educativo, a fin de informar y retroalimentar los procesos de desarrollo de la política curricular nacional” (Mineduc, 2016c, p.39).

Esta consignación en el discurso curricular, resultaría esperanzadora, porque aquellos conceptos no solo estarían aludiendo a un tipo de diversidad derivada de los pueblos originarios, sino que abarcaría la realidad migrante, aún en forma indirecta, a que día a día se hace más presente en nuestras calles y aulas. No obstante, es necesario apuntar que el uso popularizado del concepto de diversidad en los discursos educativos institucionalizados es realizada desde operaciones binarias, donde el otro, el diverso, puede ser categorizado desde "planteamientos relativistas de la diferencia cultural que amparan la equiparación de las diferencias entre grupos sociales en términos exclusivamente simbólicos sin considerar otros factores estructurales de desigualdad y exclusión” (Menéndez, 2010 en Jiménez \&Guzmán, 2013, p. 341). 
Ya en el nivel de concreción curricular de la escuela, el reconocimiento anterior tendría que avanzar hacia una vivencia de interculturalidad migrante, desde los Planes de Formación ciudadana, que cada establecimiento educacional está llamado a elaborar, bajo el mandato de la Ley de Formación Ciudadana (Ley N 20.911, 2 de abril de 2016). Esta ley impulsa la creación en todas las escuelas y liceos de un Plan de Formación Ciudadana y Derechos Humanos y además, compromete al Ministerio de Educación a presentar antes de 2017 la asignatura de Formación Ciudadana en tercero y cuarto medio. La institucionalidad ministerial ha proporcionado como insumo el texto "Orientaciones para la elaboración del Plan de Formación Ciudadana” (Mineduc,2016d ).

Esto podría constituir un segundo factor de esperanza, ya que, si bien desde la política educacional aún la escolarización demigrantes permanece invisible, cada escuela puede formular acciones de visibilización, conforme a la realidad de migración que experimenta, valorizando de paso el rol intelectual de los docentes con capacidad de pensar y diseñar soluciones pedagógicas atingentes, dejando de ser un mero técnico que implementa las políticas pensadas desde la institucionalidad ministerial. En otras palabras, y en coherencia con el sentido profundo de interculturalidad, ella está por construir (Walsh, 2010).La visibilización de la multiculturalidad migrante interpela y enriquece también al concepto de ciudadanía que se pretende desarrollar a través de la integración de la Formación ciudadana donde cada en la escuela está llamada a crearlo desde su particular contexto, lo cual podría evitar una visión homogeneizada de la migración en Chile, porque constituyen experiencias diferentes ser inmigrante en Santiago, en las regiones fronterizas o en la zonza centro sur del país.

Contradictoriamente, en el documento "Orientaciones para la elaboración del Plan de Formación Ciudadana”(Mineduc, 2016d), se observa que la alusión a la diversidad cultural más bien interpela a una noción de interculturalidad funcional (Tubino,2005; Walsch,2010) con rasgos de folclorización, ejemplificado en la sugerencia de acción referida a la organización de una "Feria intercultural”. Esta actividad se propone para los niveles desde $1^{\circ}$ a $6^{\circ}$ año de educación básica, con el objetivo que "las y los niños expongan la riqueza del patrimonio cultural de todos los pueblos originarios de nuestro país y las principales naciones migrantes" (Mineduc, 2016 d) p.55). Se deriva entonces, un tratamiento exótico y amable de la multiculturalidad (Barquín, 2015), legitimado a través de este instrumento curricular. En consecuencia, resulta imperativo tensionar el uso del concepto de diversidad cultural, tanto en la política educativa e instrumentos curriculares,que, en el caso de las personas migrantes, pareciera reducirse a una configuración esencializada y homogeneizadorade su cultura (Jiménez \&Guzmán, 2013). 


\section{Formación inicial docente}

Desde la política estatal, en el intento de orientar a las instituciones formadoras de profesores en las competencias fundamentales para ejercer un efectivo proceso de enseñanza, se formulanlos Estándares orientadores para egresados de carreras de Pedagogía en Educación Parvularia (Mineduc, 2012a), Pedagogía en Educación Básica (Mineduc,2011) y Pedagogía media (Mineduc,2012c). Ellos entregan "una orientación acerca de los conocimientos y habilidades necesarios que debería manejar el egresado de pedagogía para enseñar estas disciplinas, sobre la base del criterio de expertos” (Mineduc, 2011, Mineduc, 2012 ay b) y del conjunto de Competencias Genéricas del Proyecto Tuning Europa-América Latina (2004-2007).

Los estándares poseen la doble dimensión pedagógica y disciplinar del futuro profesor, consignando dentro de las habilidades profesionales básicas, tanto para el nivel primario y secundario, un estándar referido a que el docente "está preparado para atender la diversidad y promover la integración en el aula” (Estándar pedagógico 8, Mineduc, 2011, p.37 y Mineduc, 2012b, p.45). La descripción del estándar se estructura destacando que el profesor en formación comprende la educación como un derecho de los estudiantes y que "la diversidad es una fuente de riqueza para el desarrollo y aprendizaje de las comunidades educativas". Dentro de los seis indicadores de este estándar es posible observar dos que resultan atingentes con la realidad de la migración y los discursos y prácticas pedagógicas del docente; el primero de ellos, apunta a la disposición de respeto que debe mostrar el docente hacia sus estudiantes y familias, considerando la influencia que pueden tener sus acciones, decisiones y juicios en el desarrollo afectivo y social de los/as niños o jóvenes (Mineduc,2011), “previniendo el efecto discriminatorio que pueden tener sus propias acciones, decisiones y juicios respecto a las características personales derivadas de variables sociales, sexuales, étnicas, de apariencia física o variables de aprendizaje (Mineduc,2012b). El segundo estándar, apunta al conocimiento por parte del profesor de estrategias y de recursos pedagógicos apropiados para favorecer su integración y el desarrollo de sus fortalezas y autonomías (Mineduc, 2011, p.37; Mineduc,2012b, p. 45).

Por su parte, los Estándares para egresados de Educación Parvularia, declaran que la educadora de párvulos ha adquirido "nociones fundamentales de las ciencias sociales, las cuales le permiten una visión amplia de la realidad cultural y social”, facilitando el desarrollo de estrategias didácticas que "favorecen que las niñas y los niños conviertan el medio cultural y social en objeto de conocimiento, y comiencen a desarrollar disposiciones tempranas de ciudadanía” (Mineduc, 2012a, p.58).

Como contraparte, la evidencia investigativa es coincidente en concluir que la FID resulta deficiente en lo referido a las temáticas de educación intercultural e inclusión de alumnos inmigrantes y se hace necesaria formación inicial y continua (González, Berríos\& Buxarris, 2013; Fernández \& Gómez, 2013; Sanhueza, Carrillo \& Quintriqueo, 2014; Zapata, 2014; Sanhueza 
et al.2016), por lo tanto los docentes desarrollan una escolarización "sin haber contado con una formación previa relativa a lo intercultural, en un contexto donde llama la atención la ausencia de un marco legal institucional y de políticas públicas tendientes al "vivir juntos” (Palma,2015, p.5). Entonces, "la suerte de cada alumno depende de la iniciativa e ideología particular de cada uno de los docentes que le sea asignado" (Beach, 2011,p.69), lo cual es coincidente con Hernández (2016) quien además agrega que "las escuelas en contextos de alumnado migrante no han logrado implementar una política inclusiva que facilite la integración de los niños y niñas extranjeros. Se evidencia la ausencia de un plan de actividades concretas para eliminar la discriminación, el racismo y la xenofobia” (Hernández, 2016,p.167). Esta orfandad en que se encuentran las escuelas, y por tanto los docentes para enfrentar el desafío derivado de la diversidad cultural, (Bustos \&Gairín, 2017), coloca sentido de urgencia a la política pública, declarando que la inclusión, "sea acompañada de iniciativas gubernamentales que permitan las condiciones para su cumplimiento” (Ibid, p. 214).

En el contexto latinoamericano, la idea de educación intercultural en la formación inicial de profesores se instaló con la Educación Intercultural Bilingüe (EIB), iniciada en la década de los ochenta gracias a los movimientos reivindicatorios de los pueblos indígenas. No obstante, surgen críticas que estiman que, al considerar solo a la población indígena, excluye al resto de la sociedad (Walsh,2010; Zapata,2014) permaneciendo ausente una concepción de diversidad cultural migrante.

Como contraparte, surgen propuestas cuyo eje es un concepto ampliado de diversidad cultural y que destacan la necesidad que la formación de los profesores transite en dicha dirección y que considere el contexto global y local; situándose en "la diversidad sociocultural inmediata, que tenga presente las asimetrías existentes en el sistema educativo” (Zapata, 2014,p.224).

Este vacío en la formación del profesorado en diversidad cultural, sumado al peso colonialista de la sociedad chilena, podrían en parte explicar las acciones de discriminación ejercida por los profesores ya sea por razones étnicas o culturales (Tijoux,2013), amparadas en el supuesto que las culturas de origen del alumnado migrante suponen un déficit. En tanto, el profesorado no varía sus prácticas, a pesar de reconocer la diferencias culturales existente en sus aulas, donde predomina "un saber y conocimiento hegemónico, donde no se acepta otra lógica de conocimiento que no sea la occidental (Henríquez et al., 2016, p. 185). A su vez, la evidencia investigativa en formación inicial docente revela como nudo altamente crítico el referido a la planificación para estudiantes de diversas culturas, donde además, la concepción de diversidad cultural que portan los y las estudiantes de pedagogía se encuentra entre los factores que determinan las formas de aproximación a desarrollar prácticas más o menos inclusivas (íbid, p. 191). 


\section{Desempeño docente}

El desempeño de los docentes, tanto directivos como de aula, está condicionado a sus respectivos "Marcos” (Marco de la Buena Enseñanza, Mineduc,2003 y Marco de la Buena Dirección y el Liderazgo Escolar, Mineduc,2015), los cuales se suman al reportorio de instrumentos curriculares por competencia que bosquejan la formación inicial docente. Si bien desde el Marco para los directores, la realidad inmigrante no es señalada en forma directa, sí están llamados en ese instrumento, a ser líderes pedagógicos con una impronta de justicia social.

No obstante, los discursos de los Estándares de formación inicial y de los Marcos de desempeño para los docentes de aula y directivos, aún permanecen distantes de una formación en educación intercultural desde un contexto latinoamericano. Es decir, de una educación con claros fines éticos y políticos, donde "el reconocimiento de las diferencias culturales" permite educar para la disposición de descentrarnos y comprender que no existe una única cultura (la dominante) y que no existen culturas ni superiores ni inferiores, solo son diferentes y, en su dimensión política, una educación que "promueve un espacio en el que, como ciudadanos, con igualdad de condiciones y derechos, podamos dialogar reconociendo y respetando nuestras diferencias culturales como enriquecedoras" (Zapata,2014,p.221). En esta línea, la acción educativa trasciende a los contenidos explícitos impartidos en clase, incluyendo "los comentarios, las actitudes, las relaciones interpersonales, las formas de organización y gestión de aula” (Mirete \&Maquilpon, 2017, p.132).

En consecuencia, y debido a los crecientes escenarios educativos multiculturales, sería esperable que, dentro de las competencias a evaluar en el desempeño docente sea considerada la competencia comunicativa intercultural, la cual comprende "las habilidades cognitivas, afectivas y comportamentales que emplean los individuos culturalmente diferentes para favorecer un grado de comunicación eficaz, estableciendo relaciones apropiadas y efectivas en determinados contextos sociales y culturales" (Henríquez et al, 2016, p. 184).

\section{Romper el silencio:Profesionalidad docente e interculturalidad crítica}

Si bien no es posible desconocer los avances de los países de América Latina y el Caribe en cobertura escolar, el acceso universal a una educación de calidad aún no se alcanza (UNICEF,2015), debido a que el modeloeducativo actual ya no responde a las necesidades que la región requiere para "incorporarse a la economía del conocimiento, y que incluyen, además de los conocimientos objetivos, lógicos o racionales (conocimiento científico), otros elementos subjetivos, analógicos o emocionales, como la intuición, la sensibilidad y la expresión artística, que se pueden y 
deben aprender” (UNICEF, 2015, p.8). Por lo tanto, la región requiere de un cambio de paradigma, que brinde espacio a las epistemes del Sur, considerando nuestros procesos históricos, características culturales y de la economía de América Latina, haciendo posible "incluir lo diferente para superar la segregación y generar una nueva identidad escolar asociada con su función cultural y no meramente disciplinadora de poblaciones potencialmente riesgosas” (Tiramonti,2011 citado en UNESCO 2015, p.26).

Esta reflexión adhiere a la idea que entiende el cambio de paradigma desde su emergencia de imperativos internos; es decir, desde los propios actores y la legitimación de sus saberes (Ferrada,Villena\&Turra,2015; UNESCO,2015), dejandoatrás la práctica de adoptar acríticamente soluciones creadas para otras realidades, lo cual genera tensiones de gobernabilidad, como es el caso de la gestión de las actuales reformas educativas que con el fin de "mejorar la eficiencia y la calidad pocas veces buscan respuestas inspiradas en la realidad y las características de la región” (UNICEF, 2015, p.45).

En esta línea, se respalda la tesis del "silencio elocuente" que vertebra este artículo, relacionada con la ausencia en Chile de una política educativa de atención a migrantes, en el sentido que dicho silencio nos habla, nos interpela y abre una doble posibilidad: por un lado, de poner en valor las experiencias de centros educativosque escolarizan a migrantes y por otra, constituye una posibilidad de actuación del docente como un intelectual capaz de pensar y diseñar con otros respuestas educativas contextualizadas a la diversidad cultural propia de su desempeño construyendo su actuar no solo a un rol técnico que aplica la política educativa gubernamental, sino más bien desde una profesionalidad docente encaminada al desarrollo de una educación intercultural crítica.

Más arriba decíamos, que en cuanto a la formación entregada por las facultades de educación a los estudiantes de pedagogía, no considera la heterogeneidad cultural de los contextos de desempeño, situación que aparece como una de las demandas de los profesores en ejercicio a la formación inicial docente en Chile (Gaete, Gómez \& Bascopé, p.2016) y también como un requerimiento en la formación continua (Mineduc,2014). Por lo tanto, resulta sugerente la pregunta que se formula Cuenca (2007) ¿hacia dónde va la formación docente en América Latina?

Sucintamente se puede responder que dicho tránsito ha estado fuertemente ligado a la profesionalización del profesorado en términos de competencias que buscan mejorar los conocimientos y las habilidades y los estándares de calidad en su práctica. No obstante, este esfuerzo por fomentar el profesionalismo, no entrega respuestas para enfrentar los nuevos escenarios educativos que exigen al docente un radio de actuación que sobrepasa el aula. Al mismo tiempo, en la gran mayoría de los países de la región, existe una desvalorización de la profesión docente (Tenti, 2005) que deriva entre otros factores, de encasillar "el papel y desempeño del docente exclusivamente en relación con la tarea pedagógico-educativa, dentro o 
fuera del aula, lo deja en una situación pasiva respecto a la gestión y a la política” (Cuenca,2007, p.26).Entonces, y a propósito de la diversidad cultural migrante, se propone la participación de los maestros en la gestión de políticas educativas como una estrategia para elevar el estatus de la profesión y transitar desde un enfoque profesionalizante a uno cuyo centro busca recuperar la profesionalidad docente. Es decir, el mejoramiento del prestigio y de la posición social (Cuenca,2007), que las reformas educativas han colaborado en mermar - al no confiar en el liderazgo intelectual y moral de los docentes, quienesquedan reducidos a la categoría de técnicos superiores- se constituye en una verdadera proletarización del trabajo del profesor, el cual asume el rol de gestionar y cumplimentar programas en lugar de asumirlos críticamente (Giroux,2001).

La elocuencia de la ausenciade una política enfocada a la escolarización de niños y jóvenes migrantes interpela también al concepto de interculturalidad, el cual fue posible leer en los discursos de los instrumentos legales y del currículo que norman la educación en Chile. En ellos se encuentran trazas de una concepción funcional de interculturalidad, como es del caso de la reciente Política de Formación Ciudadana (Mineduc,2016 c), referida en apartados anteriores. El interculturalismo funcional releva el diálogo y el reconocimiento entre culturas, pero invisibiliza la condición de pobreza en las cuales viven los integrantes de las culturas subalternas, por lo tanto "no cuestiona el sistema postcolonial vigente y facilita su reproducción.

El concepto funcional (neoliberal) de interculturalidad genera un discurso y una praxis legitimadora que se viabiliza a través de los Estados nacionales, las instituciones de la sociedad civil” (Tubino, 2005, p.6). Por su parte Walsh indica este hecho como una "moda", en sentido crítico, al develar que las reformas educativas de la década del noventa, siguiendo la lógica del capitalismo transnacional (Walsh, 2010, p. 5) de ese tiempo y las políticas que emergen en este nuevo siglo, entiende lo intercultural como "parte del aparato de control y de la política educativa estatal” (Walsh,2010, p. 81), que más bien profundiza las desigualdades y deja intactas la estructuras de poder; es decir, es una interculturalidad funcional al sistema.

Por el contrario, la propuesta de la interculturalidad crítica aspira a la construcción de otros modos del poder, saber, ser y vivir, más allá de las actuales expresiones de educación intercultural bilingüe (Walsh,2010) o de la relación entre los diferentes grupos culturales, sino que aboga por "visibilizar, enfrentar y transformar las estructuras e instituciones que diferenciadamente posicionan grupos, prácticas y pensamientos dentro de un orden y lógica que, a la vez y todavía, es racial, moderno-occidental y colonial” (Walsh,2010, pp.91-92). Por lo tanto, Walsh junto con conceptualizar la interculturalidad crítica como un proyecto, un proceso y una lucha de tipo política, social, epistémica y ética, también la consiga como una herramienta pedagógica, que "pone en cuestionamiento continuo la racialización, subalternización e inferiorización y sus patrones de poder, visibiliza maneras distintas de ser, vivir y saber, y busca el desarrollo 
y creación de comprensiones y condiciones que no solo articulan y hacen dialogar diferencias en un marco de legitimidad, dignidad, igualdad, equidad y respeto, sino también-y a la vez-alientan la creación de modos “otros” de pensar, ser, estar, aprender, enseñar, soñar y vivir que cruzan fronteras” (Walsh, 2010, p. 92).

Los procesos migratorios transnacionales que viene experimentado Chile apelan a una nueva forma de comprender la educación, la educación intercultural, que implica "el encuentro de toda la sociedad en el marco de un entorno común de intercambio cultural y no exclusivamente a las minorías étnicas e inmigrantes” (García-Yepes, 2017, p190), donde la escuela, a través del currículum, deconstruya las representaciones etnocéntricas de la cultura "para generar un aprendizaje basado en la existencia compartida de representaciones culturales” (íbid,p.198). Si bien una de las dimensiones más complejas de abordar es plasmar la diversidad cultural en el currículum, debido a que implica asumir desde una dimensión política el proceso educativo, en términos de relaciones de poder, reconociendo las desigualdades e inequidades derivadas de la posición en que se encuentran los/as alumnos/ as migrantes en la escuela (Jiménez, Aguilera, Valdés y Hernández, 2017), a la perspectiva intercultural, como proceso integrador, da fuerza a la necesidad de "romper con las nociones hegemónicas y totalitarias que se han construido en torno a la cultura, la nación y la diversidad” (López \&Cuello, 2016, p.374).

En definitiva, la gestión de la diversidad cultural migrante alude a una cuestión de saberes, donde la discusión transita entorno hacia lo "que realmente queremos saber lo que sabemos o se transmite como lo que hay que saber hoy, sino también de la prioridad en la transmisión del saber que a la humanidad le importa conocer" (Fornet-Betancourt, 2006, p.40). El resultado de dicha discusión, queda plasmado en el currículum escolar, específicamente en el instrumento de los programas educativos, que deberían recoger la diversidad de cosmovisiones del género humanos, y no el proyecto civilizatorio hegemónico.

Es necesario señalar que el componente interculturalidad está siendo visto por las universidades como un vacíoen la formación de profesionales de distintas áreas, y como ejemplo de una de las competencias menos desarrolladas y más necesarias debido a los crecientes escenarios diversos culturalmente. Por lo tanto, la formación en interculturalidad y migraciones debiese estar presente tanto como una competencia profesional como un rasgo de profesionalidad docente.

A la luz de lo expuesto, se confirma que para llegar a construir un sistema educativo sólido desde una perspectiva intercultural, se requiere un trabajo en dos vías paralelas. La primera consiste en gestionar políticas educativas atingentes al nivel de desarrollo de las capacidades instaladas en el sistema educativo. La segunda vía apunta a construir las capacidades que abrirán las puertas para la siguiente etapa de desarrollo educativo del país. En otras palabras, se debe gestionar efectivamente el sistema educati- 
vo actual a la vez que se toman medidas para construir las capacidades que permitirán avanzar hacia la siguiente fase de desarrollo” (UNESCO,2016, p.142).

Si bien en los desafíos y recomendaciones de política educativa a partir del TERCE, no se observa una alusión directa a la escolarización de migrantes, si resultaría atingente considerar el tipo de implementación de abajo hacia arriba, que "apunta a reconocer la capacidad de los actores locales tanto para implementar y ajustar programas nacionales a la realidad local, como para desarrollar intervenciones propias específicas que pueden estar alineadas con las políticas nacionales”, sin olvidar las condiciones de desigualdad socioeconómica y la pobreza, que afecta aún a importantes sectores de la población, son dos atributos del contexto que deben considerarse al momento de diseñar políticas educativas” (UNESCO,2016, p.141).

\section{Conclusiones}

En Latinoamérica las políticas educativas interculturalesestán focalizadas en la diversidad cultural indígena (Mondaca \& Gajardo, 2013), situación de la cual Chile no está exento, a lo cual suma una legislación migratoria obsoleta que concibe al extranjero como una amenaza. Las políticas educativas responden a un modelo de Estado-Nación unitario y homogéneo, que se autodenominan multiculturales, pero más bien tienden a estigmatizar al otro, al diferente, no poniendo en relación las diferentes culturas de un territorio determinado. Este silencio institucional pone al Estado chileno en una situación de entredicho, porque no está dando cumplimiento a los acuerdos internacionales que ha firmado, los cuales buscan resguardar los derechos de las personas, especialmente niñas y niñas, en situación de migración. En palabras simples, el modo indiferente en que el Estado de Chile está recibiendo a los y las estudiantes migrantes, revela una negación de la diversidad cultural, coherente con los afanes homogeneizadores y de reproducción de la cultura dominante que la educación ejerce a través de la escuela. La ausencia de una política educativa para migrantes implica no reconocer la presencia del otro, y si no se le reconoce, entonces, no se le respeta; de lo cual se deriva la invisibilización de sus saberes identitarios en el currículum.

A nivel curricular, los peligros que la institucionalidad no entregue una respuesta a los desafíos que entraña la diversidad cultural de vertiente migratoria radican en la profundización de un racismo de tipo cultural, donde subyace la concepción estática de cultura, "un corpus de características inamovibles que clasifican a los grupos humanos y cuya distancia entre ellas hace que algunas culturas (por atrasadas, por su fanatismo religioso, por su lejanía local...) sean inintegrables en la sociedad receptora, además de un peligro para la pureza de ésta ( Delgado, 1998 en Barquín, 2015, p. 455). Al contrario, el ejercicio necesario es abrir al mundo el currículum escolar, indistintamente si existen o no estudiantes migrantes (Barquín, 2015) y avanzar del estado actual de visibilización de la diversidad cultural migrante (Moreno \&Oyarzún, 2013) hacia la reinvidicación de sus formas 
de pensar y conocer. Al respecto se estima que la elocuencia del silencio derivado de la ausencia de una política de escolarización de niños y jóvenes migrantes puede constituirse en una oportunidad para fortalecer procesos de profesionalidad docente, considerando sus saberes en el diseño de dicha política por construir. El tránsito hacia la profesionalidad conlleva a repensar la naturaleza del trabajo y el rol del docente como un intelectual, implica considerar la base teórica e intelectual de su tarea (Mora, 2004). En esta línea, la evidencia investigativa nacional documenta como demanda que los procesos formativos del profesorado den un giro hacia la profesionalidad, incluyendo "la preparación de docentes críticos, con pensamiento reflexivo, sistémico y autónomo, capaces de justificar y hacer valer sus decisiones profesionales” (Gaete, Gómez \& Bascopé 2016, p.13; Ferrada, Villena \&Turra,2015).

A su vez, este silencio, representa también un desafío político educativo necesario de asumir en la atención a la diversidad cultural, que permitiría soslayar la impronta funcional de interculturalidad que se desprende del análisis del marco curricular vigente, y a su vez, abriría caminos donde el horizonte sea la educación intercultural crítica, a través del desarrollo depropuestas de escolarización de migrantes bajo la modalidad de abajo hacia arriba. Es decir, los actores educativos deben permanecer vigilantes, cuestionando el uso del concepto diversidad frente a la amenaza de miradas reduccionistas frente a la diversidad de culturas que bullen en las aulas. Al respeto, es la perspectiva intercultural crítica quien hace patente "el problema estructural-colonial-racial y su ligación al capitalismo de mercado" (Walsh, 2012, p.65) y que también promueve el diálogo simétrico de las epistemologías y el combate de la exclusión curricular y epistemológica, donde los programas educativos "pueden ser espacios de participación en los que se discierne interculturalmente qué es lo que debemos saber y transmitir para la universalización humanizadora de cada ser humano" (FornetBetancourt,2006, p. 40).

La situación actual revela una inconsistencia de los discursos legales y curriculares que garantizan el acceso a la educación, pero no las condiciones de permanencia del alumnado extranjero. Entonces, el proceso de escolarización de estudiantes migrantes es abordado desde la política educativa en forma insuficiente, bajo el principio del acceso al sistema escolar. En cuanto a los instrumentos curriculares analizados, resultan ser cómplices de un silencio, porque el niño o joven es formado desde una perspectiva asimilacionista, persistiendo aún un enfoque de integración que dista del horizonte trazado desde el enfoque inclusivo. Además, en dichos instrumentos, las culturas migrantes permanecen subsumidas en la categoría “diversidad cultural”, uso retórico que adolece de especificidad, porque que alude también a otros tipos de diversidades. Esta ausencia en el currículum, nos dice que la diversidad cultural migrante no ha sido objeto de reflexión sistematizada por lo actores involucrados, viniendo a reforzar la existencia de un Estado unitario, donde bajo una concepción de igualdad mal entendida, camufla un enfoque asimilacionista y que legitima la matriz monocultural que reproduce la escuela a través del currículo. 


\section{Agradecimientos}

Este texto fue presentado como ponencia en el Simposio Internacional Dirección, Gestión, Liderazgo y Política Educativa, organizado por la Red Iberoamericana de Pedagogía (REDIPE) y la Universidad Católica del Maule. Talca, Chile, 6 y 7 de abril de 2017. 


\section{Nota}

${ }^{1}$ Si vas para Chile es el nombre del vals compuesto en 1942 por Enrique Motto Arenas, más conocido como Chito Faró. Si se pone atención en la letra, ella trata de un chileno que está fuera de país, es decir, es un migrante, que le pide a un viajero que viene a Chile, que pase por donde su amada, que vive en una "casita muy linda y chiquita que está en la falda de un cerro enclavada” y que, además, le diga que se muere de amor por ella. 


\section{Bibliografía}

Arias, M. (2016). \#NoMásRut100: la campaña por los derechos de los alumnos migrantes en Chile. Recuperado de_ http:// www.eligeeducar.cl/nomasrut100-campana-derechos-alumnosmigrantes

Barquín, A. (2015). ¿Qué debe hacer la escuela con las culturas familiares del alumnado inmigrante? Educar, 51(2). 443-464. Recuperado de evistes.uab.cat/educar2/article/view/v51-n2-barquin/671-pdf-es

Beech, J. y Princz, P. (2012). Migraciones y educación en la Ciudad de Buenos Aires: tensiones políticas, pedagógicas y étnicas. Revista Latinoamericana de Inclusión Educativa, 6(1), 53-71. Recuperado de http://www.rinace.net/rlei/numeros/vol6-num1/art3.pdf

Bustos, R y Gairín, J. (2017). Adaptación académica de estudiantes migrantes en contextos de frontera. Revista Calidad en la Educación. 46, 193220.doi: 10.4067/S0718-45652017000100193

Congreso de Chile (14 de agosto de 1990). Promulga Convención sobre los Derechos del niño (Decreto Supremo No830). Recuperado de: https:/ /www.leychile.cl/Navegar?idNorma=15824_

Congreso de Chile (17 de agosto del 2009). Ley General de Educación. (Ley 20.370 de 2009). Recuperado de: http://www.leychile.cl/ Navegar?idNorma $=1014974$

Congreso de Chile (29 de mayo de 2015) Ley de Inclusión escolar que regula la admisión de los y las estudiantes, elimina el financiamiento compartido y prohíbe el lucro en establecimientos educaciones que reciben aportes del estado. (Ley 20.845 de 2015). Recuperado de: https://www.leychile.cl/Navegar?idNorma=1078172

Cuenca, R., Nucinkis, N. y Zabala, V. (comps.) (2007). Nuevos maestros para América Latina. Madrid: Morata.

Donoso, S. y Donoso, G. (2009). Políticas de gestión de la educación pública escolar en Chile (1990-2010): una evaluación inicial”. Ensaio: aval. Pol.públ. Educ., Río de Janeiro, 17(64), 421-448. Recuperado de http://www.scielo.br/pdf/ensaio/v17n64/v17n64a03.pdf

Faró, Ch. (1942). Si vas para Chile. Versión de "Los cuatro cuartos”,1991, Sello Columbia.

Fernández, T. y Gómez, P.(2013).El programa Malted: una propuesta didáctica para enseñar español a inmigrantes en la escuela primaria en España.Actualidades Investigativas en Educación, 13(2). 54-86 DOI: https://doi.org/10.15517/aie.v13i2.11736 
Ferrada, D., Villena, A \& Turra, O. (2015). Transformar la formación. Las voces del profesorado. Santiago: RIL Editores.

Fornet-Betancourt, R. (2006). La interculturalidad a prueba. Recuperado de http://www.uca.edu.sv/filosofia/admin/files/1210106845.pdf

Gaete, A., Gómez, V. \& Bascopé, M. (2016). ¿Qué le piden los profesores a la formación inicial docente en Chile?, Centro de Políticas Públicas UC, Temas de la Agenda Pública, Santiago, 11(86). Recuperado de: http://politicaspublicas.uc.cl/wp-content/uploads/2016/06/ Qu\%C3\%A9-le-piden-los-profesores-a-la-formaci\%C3\%B3n-inicialdocente-en-Chile.pdf

García-Yepes, K. (2017). (2017). Procesos de integración social de inmigrantes en escuelas de Huelva, España. Alteridad, 12(2), 188200. doi: 10.17163/alt.v12n1.2017.05

Giroux, H. (2001). Los profesores como intelectuales transformativos. Docencia, 6(15), 60-66.

González, J. (1987). “Maldito sudaca”. La cultura de la basura, Santiago: Emi-Odeón.

González, O., Berríos, Ll. y Buxarrais, M. (2013). La sensibilidad del profesorado hacia el modelo de educación intercultural: Necesidades, situación actual y propuesta de un instrumento de medida.Estudios pedagógicos, 39(2), 147-164. Recuperado de: http://dx.doi.org/ $10.4067 /$ S0718-07052013000200010

Hernández, A. (2016). El currículo en contextos de estudiantes migrantes: Las complejidades del desarrollo curricular desde la perspectiva de los docentes de aula”. Estudios Pedagógicos. XLII (2), 151-169. Recuperado de: http://www.scielo.cl/pdf/estped/v42n2/art09.pdf

Hevia, R. (coord.). (2005). Políticas educativas de atención a la diversidad cultural. Brasil, Chile, Colombia, México y Perú. Santiago: Oficina Regional de Educación de la UNESCO para América Latina y el Caribe OREALC/UNESCO.

Jiménez, M. y Guzmán, R. (2013). Sociología de la construcción de los conceptos académicos: el caso de la “diversidad” en educación. Sociología histórica, 2, 321-353. Recuperado de: http://revistas.um.es/ sh/article/viewFile/189021/155681

Jiménez, F., Aguilera, M., Valdés, R., Hernández, M. (2017). Migración y escuela: análisis documental en torno a la incorporación de inmigrantes al sistema educativo chileno. Psicoperspectivas. Individuo y Sociedad, 16(1), 105-116. doi:10.5027/psicoperspectivas-vol16-issue1fulltext-940 
López, A. y Cuello, E. (2016). La educación intra e intercultural como enfoque pedagógico “propio”. Revista Colombiana de Ciencias Sociales, 7(2), 370-387. doi: 10.21501/22161201.1780

Ministerio de Educación, Gobierno de Chile. (2010). Ley General de Educación (Ley N² 20.370, 17 de agosto del 2009). Disponible en: http:/ /www.leychile.cl/Navegar?idNorma=1014974

Ministerio de Educación, Gobierno de Chile. (2003). Marco para la Buena Enseñanza. Recuperado de: http://portales.mineduc.cl/usuarios/ cpeip/File/Documentos\%202011/MBE2008.pdf

Ministerio de Educación, Gobierno de Chile. (2005). Bases curriculares de la Educación Parvularia. Recuperado de: http://portales.mineduc.cl/ usuarios/convivencia_escolar/doc/201103041242340.Bases_ Curriculares_de_Educacion_Parvularia_2001.pdf

Ministerio de Educación, Gobierno de Chile. (2011). Estándares orientadores para egresados de carreras de Pedagogía en Educación Básica. Recuperado de: http://ww2.educarchile.cl/UserFiles/P0001/Image/ Estudiantes_pedagogia/estud_pedagogia/estandares_Fid/ estandar_basica.pdf

Ministerio de Educación, Gobierno de Chile. (2012a)). Estándares orientadores para egresados de carreras de Pedagogía en Educación Parvularia. Recuperado de: http://portales.mineduc.cl/usuarios/ cpeip/doc/201205111816290.LibroEstandaresOrientadores CarrerasEducaciOnParvulariaconcarta.pdf

Ministerio de Educación, Gobierno de Chile. (2012 b)). Bases curriculares para la Educación Básica. Recuperado de: http:// www.curriculumenlineamineduc.cl/ 605 / articles 30013_recurso_14.pdf

Ministerio de Educación, Gobierno de Chile. (2012 c)). Estándares orientadores para egresados de carreras de Pedagogía Media. Recuperado de: http://portales.mineduc.cl/usuarios/cpeip/File/ librosestandaresvale/libromediafinal.pdf

Ministerio de Educación, Gobierno de Chile. (2014). Encuesta Internacional de Enseñanza y Aprendizaje (TALIS). OECD. Recuperado de: http://centroestudios.mineduc.cl/tp_enlaces/portales/ t p 5996 f 8 b 7 c m 96 / u pload Im g/File/Evidencias / evidencia_final_marzo_2015.pdf

Ministerio de Educación, Gobierno de Chile. (2015). Marco para la Buena Dirección y Liderazgo. Recuperado de: http://portales.mineduc.cl/ usuarios/cpeip/doc/201511131613560.MBD\&LE_2015.pdf 
Ministerio de Educación, Gobierno de Chile. (2016 a)). Mineduc anuncia la creación del Identificador Provisorio Escolar IPE para estudiantes migrantes. Recuperado de http://www.mineduc.cl/2016/11/21/ mineduc-anuncia-la-creacion-del-identificador-provisorio-escolar-ipeestudiantes-migrantes/

Ministerio de Educación, Gobierno de Chile. (2016 b)).Orientaciones para la construcción de comunidades educativas inclusivas.Ministerio de Educación, División de Educación General Coordinación Nacional de Inclusión y Diversidad. Recuperado de: http://www.mineduc.cl/wpcontent/uploads/sites/19/2017/03/Documento-Orientaciones28.12.16.pdf

Ministerio de Educación, Gobierno de Chile. (2016 c)). Recomendaciones para una Política Nacional de Desarrollo Curricular. Informe Mesa de Desarrollo Curricular. Unidad de Currículum y Evaluación. Ministerio de Educación de Chile. Recuperado de: http:// www.curriculumenlineamineduc.cl/605/articles-35514_recurso_1.pdf

Ministerio de Educación. Gobierno de Chile. (2016 d)). Orientaciones para la elaboración del Plan de Formación Ciudadana. Recuperado de: http://www.mineduc.cl/wp-content/uploads/sites/19/2016/04/ OrientacionesPFC.pdf

Ministerio de Justicia (1998). Derechos. Santiago: Ministerio de Justicia y Ministerio de Planificación y Cooperación,

Mirete, A. y Maquilón, J. (2017). Estado de conciencia de los docentes hacia la diversidad cultural. Un análisis por dimensiones. INTERACÇÕES. 43,116-134. Recuperado de: http://revistas.rcaap.pt/ interaccoes/article/view/12034/9163

Mondaca, C. yGajardo, Y. (2013). La educación intercultural bilingüe en la región de Arica y Parinacota, 1980-2010. Diálogo Andino, 42, 69-87. Recuperado de:

Mora, L. (2004). Ser profesor hoy: desde la paradoja hacia una nueva profesionalidad. Revista Horizontes Educacionales. Facultad de Educación y Humanidades. Universidad del Bío-Bío, 9, 57-63.

Moreno, K. y Oyarzún, C. (2013). ¿Interculturalidad en el sistema educacional chileno?: representaciones sociales de la integración de estudiantes extranjeros en dos establecimientos educacionales municipales. Revista Electrónica de Psicología Iztacala, 16(3), 764-790. Recuperado de: http://www.revistas.unam.mx/index.php/repi/article/ view/41789/37949

Oliva, M.A. (2008). Política educativa y profundización de la desigualdad en Chile”. y Quintriqueo, S. (2014). Estudio exploratorio sobre las 
actitudes y comportamiento del profesorado de Chile en contextos de escolarización de alumnado inmigrante. Revista electrónica de investigación educativa, 16(3) 148-162. Recuperado de: http:// redie.uabc.mx/vol16no3/contenido-sanhueza-friz-.html

Organización de los Estados Americanos. (2011). Educación para niñas, niños y jóvenes inmigrantes en las Américas: Situación actual y desafíos. Recuperado de: http://www.oas.org/es/sadye/publicaciones/ educacion-inmigrantes.pdf

Palma, M. (2015). Inclusión de los niños inmigrantes: prácticas sociales en el espacio educativo. En N. Garita (Presidencia).Acta académica. XXX Congreso de la Asociación Latinoamericana de Sociología,San José, Costa Rica. Recuperado de: http://sociologia-alas.org/congreso-xxx/ponencias

RAE (2014). Diccionario de la Lengua Española. Recuperado de: http:// dle.rae.es/?id=EYQIg19

Sandoval, R. (2016). Hacia una Política Nacional Migratoria. En Tijoux, M.E. (Ed.). Racismo en Chile. La piel como marca de la inmigración (pp.103-112). Santiago: Ed. Universitaria.

Sanhueza, S.; Friz, M. y Quintriqueo, S. (2014). Estudio exploratorio sobre las actitudes y comportamiento del profesorado de Chile en contextos de escolarización de alumnado inmigrante. Revista Electrónica de Investigación Educativa, 16(3), 148-162, 148-162. Recuperado de http://redie.uabc.mx/vol16no3/contenido-sanhueza-friz-.html

Sanhueza,S., Patrick. P., Hsuc, Ch., Domínguez, J., Friz, M. y Quintriqueo, S. (2016). Competencias Comunicativas Interculturales en la formación inicial docente: El caso de tres universidades regionales de Chile. Estudios Pedagógicos, XLII( 4) 183-200. http://dx.doi.org/10.4067/ S0718-07052016000500011

Stefoni, C, Acosta, E, Gaymer, M.y Casas-Cordero, F. (2008). Niños y niñas inmigrantes en Santiago de Chile: entre la integración y la exclusión. Chile: Universidad Alberto Hurtado y OIM.

Thayer, L. (2016). Migrantes en Chile. Una aproximación a su condición social y a algunos elementos para entender la subjetividad. En Erazo, X; Esponda, J. y Yaksic, M. (Ed). Migraciones y derechos humanos: mediación social intercultural en el ámbito local (pp. 71-98). Santiago: LOM.

Tenti, E. (2005). La condición docente. Análisis comparado de la Argentina, Brasil, Perú y Uruguay. Argentina: Siglo XXI Editores. 
Tijoux, M. (2013). Las escuelas de la inmigración en la ciudad de Santiago: elementos para una educación contra el racismo. Polis, Revista Latinoamericana, 35(12), 287-307. Recuperado de http://www.scielo.cl/ pdf/polis/v12n35/art13.pdf

Torres, J. (2012). La justicia curricular. El caballo de Troya de la cultura escolar. Madrid: Ed. Morata.

Tubino, F. (2005). La interculturalidad crítica como proyecto ético-político, en Encuentro continental de educadores agustinos, Lima. Recuperado de: http://oala.villanova.edu/congresos/educación/lima-ponen02.htm

UNESCO (2016). Recomendaciones de Políticas Educativas en América Latina en base al TERCE. Santiago: Oficina Regional de Educación para América Latina y el Caribe, OREALC/UNESCO. Recuperado de: http://www.unesco.org/new/fileadmin/MULTIMEDIA/FIELD/ Santiago/pdf/Recomendaciones-politicas-educativas-TERCE.pdf

UNICEF (2015). El aprendizaje bajo la lupa: Nuevas perspectivas para América Latina y el Caribe. Panamá: Oficina Regional para América Latina y el Caribe. Recuperado de: https://www.unicef.org/lac/ UNICEF_Aprendizaje_bajo_la_lupa_nov2015(1).pdf

Universidad Diego Portales (2017). Informe anual sobre Derechos Humanos en Chile 2017. Santiago:

Walsh. C. (2010). Interculturalidad crítica y educación intercultural”. En Viaña, J., Tapia, L. y Walsh, C. Construyendo Interculturalidad Crítica. (pp.75-96) Bolivia: Instituto Internacional de Integración del Convenio Andrés Bello. Recuperado de: http://aulaintercultural.org/ 2010/12/14/interculturalidad-critica-y-educacion-intercultural/

Walsh. C. (2012). Interculturalidad y (de)colonialidad: Perspectivas críticas y políticas. Visão Global, Joaçaba, (15)1-2, 61-74. Recuperado de https://editora.unoesc.edu.br/index.php/visaoglobal/article/download/ $3412 / 1511$

Walsh, C. (En prensa). Interculturalidad crítica y pedagogía de-colonial: Insurgir, re-existir y revivir. En Candau V. (Edit.). Educação Intercultural hoje en América latina: concepções, tensões e propostas. Brasil: Recuperado de: https://www.maxwell.vrac.puc-rio.br/13582/ 13582.PDFXXvmi=di9ixOJob3xjBuscxZPZhgoEsplxlhlzBvSzkD ZvGWPisju2il2ACqe6QB4dTpMuWG5hj5O12FjvZPzhbBRN27TTJZ7 DpU9mBeEVjtWm9hT97srj4k77s21c28Qr30OEetBzxxV8PQ ixjKho5pvxswLuGCWJCMoRg0rfzoopfHnzrGxx6t7z2S5 Iv2r9OR26RtlrSKR8rfHUWGt3bBDduIW1LpaBhcHaH3CAOtIhKqdH 8HLjjoxO8uQUV2vdfdoW 
Zapata. R. (2014). Diversidad cultural en la formación del futuro profesorado en América Latina. Necesidades y perspectivas. Revista Electrónica Interuniversitaria de Formación del Profesorado, 17(2), 219234. doi: http://dx.doi.org/10.6018/reifop.17.2.197611

Recibido: 09.03.17

Aceptado: 09.03.17 